\title{
A morphological comparison between giant radio halos and radio mini-halos in galaxy clusters
}

\author{
R. Cassano ${ }^{1}$, M. Gitti ${ }^{2}$, and G. Brunetti ${ }^{1}$ \\ 1 INAF - Istituto di Radioastronomia, via P. Gobetti 101, 40129 Bologna, Italy \\ e-mail: rcassano@ira.inaf.it \\ 2 INAF - Osservatorio Astronomico di Bologna, via Ranzani 1, 40127 Bologna, Italy
}

Received 12 May 2008 / Accepted 9 June 2008

ABSTRACT

\begin{abstract}
In this letter we present a morphological comparison between giant radio halos and radio mini-halos in galaxy clusters based on radio-X-ray luminosity, $P_{1.4}-L_{\mathrm{X}}$, and radio luminosity-size, $P_{1.4}-R_{\mathrm{H}}$, correlations. We report evidence that $P_{1.4}-L_{\mathrm{X}}$ and $P_{1.4}-R_{\mathrm{H}}$ trends may also exist for mini-halos: mini-halo clusters share the same region of giant halo clusters in the $\left(P_{1.4}, L_{\mathrm{X}}\right)$ plane, whereas they are clearly separated in the $\left(P_{1.4}, R_{\mathrm{H}}\right)$ plane. The synchrotron emissivity of mini-halos is found to be more than 50 times larger than that of giant halos, implying a very efficient process for their origins. By assuming a scenario of sporadical turbulent particle re-acceleration for both giant and mini halos, we discuss basic physical differences between these sources. Regardless of the origin of the turbulence, a more efficient source of injection of particles, which eventually takes part in the re-acceleration process, is required in mini-halos, and this may result from the central radio galaxy or from proton-proton collisions in the dense cool core regions.
\end{abstract}

Key words. radiation mechanism: non-thermal - galaxies: clusters: general - radio continuum: general - X-rays: general

\section{Introduction}

The intra-cluster medium (ICM) consists not only of hot gas emitting in X-rays but also of non-thermal components. The major evidence for this comes from observations in the radio band where Mpc-scale diffuse synchrotron emission from the ICM is detected in a number of clusters (e.g. Feretti 2005; Ferrari et al. 2008), indicating the presence of relativistic electrons and magnetic fields. These radio sources are generally referred to as giant radio halos when located at the cluster center and radio relics when located at the cluster periphery. There are also some examples of diffuse radio emission on smaller scales ( 200-500 kpc), referred to as mini radio halos, extending around powerful radio galaxies at the center of some cool core clusters, i.e., clusters characterized by a very peaked surface brightness profile and short central cooling time formerly known as "cooling flow" clusters (e.g. Peterson \& Fabian 2006). Galaxy clusters hosting giant halos are found to always be characterized by a peculiar dynamical status indicative of very recent or ongoing merger events (e.g., Buote 2001; Schuecker et al. 2001), whereas clusters hosting mini halos are characterized by a cool core, with or without signs of moderate dynamical activity. Several statistical studies reveal that radio halos are not common in clusters (Giovannini et al. 1999; Kempner \& Sarazin 2001; Venturi et al. 2008; Brunetti et al. 2007; Cassano et al. 2008); instead, the statistics for mini halos is much poorer.

The main difficulty in understanding the origin of the synchrotron emitting electrons in both giant halos and mini halos is related to the fact that the diffusion length of the relativistic electrons is much shorter than the typical scale of the radio emission (e.g., Brunetti 2003). Therefore both giant halos and mini halos cannot be explained in terms of simple diffusion of the relativistic electrons from one or more cluster radio galaxies.
Two main possibilities have been proposed so far to explain the origin of both giant radio halos and mini radio halos: $i$ ) reacceleration models, whereby relativistic electrons injected in the ICM are re-energized in situ by turbulence. Turbulence in radio halos is supposed to be generated by massive merger events (e.g., Brunetti et al. 2001; Petrosian et al. 2001). In mini radio halos, a seed large-scale turbulence frozen into the flow could be amplified by the compression of the ICM in the cool core (Gitti et al. 2002, 2004); ii) secondary electron models, whereby the relativistic electrons are secondary products of the hadronic interactions of cosmic rays (CR) with the ICM (e.g., Blasi \& Colafrancesco 1999, for giant halos; Pfrommer \& Enßlin 2004; Fujita et al. 2007, for mini-halos). Although the properties of giant halos and mini halos are clearly different (different size, different dynamical state of the hosting clusters), it is not clear whether they are different astrophysical phenomena or if they might share similar physics. In this letter we carry out a morphological comparison between giant radio halos and mini radio halos aimed at studying the differences between their physical properties. We also consider the case of diffuse cluster sources with intermediate properties between giant halos and mini halos. To do this we investigate the presence of scaling relations between the main properties of these sources. A $\Lambda$ CDM cosmol$\operatorname{ogy}\left(H_{0}=70 \mathrm{~km} \mathrm{~s}^{-1} \mathrm{Mpc}^{-1}, \Omega_{\mathrm{m}}=0.3, \Omega_{\Lambda}=0.7\right)$ is adopted.

\section{Selection of clusters with diffuse radio emission}

We collect from the literature all clusters with well-studied giant radio halos and mini radio halos, as defined in the following.

- Giant radio halos (hereafter GHs): radio sources typically extended on Mpc scales found at the center of non-cool core clusters. We consider as belonging to this class all the 
diffuse radio sources larger than the core radius of the cluster. This is an important physical information relating the origin of these sources to physical processes taking place on cluster scales. We end up with $19 \mathrm{GHs}, 15$ of which have already been analyzed in Cassano et al. (2007, hereafter C07), 3 come from a recent survey carried out with the GMRT: A 209, A 697 and RXCJ 2003.5-2323 (Venturi et al. 2008), and 1 is a smaller halo found in A 3562 (Venturi et al. 2003; Giacintucci et al. 2005), which extends however beyond the cluster core. The presence of recent or ongoing merger events is well-established in all clusters of galaxies hosting these GHs.

- Mini radio halos (hereafter MHs): radio sources with total size $\sim 140-500 h_{70}^{-1} \mathrm{kpc}$ surrounding the central radio galaxy at the center of cool core clusters (hereafter CCCs; Table 1). Their size is comparable to that of the cooling region and smaller than the cluster core. For a long time, clusters hosting MHs have been identified with relaxed clusters based on the presence of cool cores at their center; however, signs of minor mergers and/or accretion of small subclumps have recently been detected in 5 clusters with MHs thanks to Chandra and XMM-Newton observations (Perseus: Churazov et al. 2004; A 2626: Wong et al. 2008; RX J1347.5-1145: Allen et al. 2002; Gitti \& Schindler 2004, Gitti et al. 2007a,b; A 2390: Allen et al. 2001; Z 7160: Mazzotta \& Giacintucci 2008).

For completeness we also consider the case of smaller scale radio sources in some merging clusters: A 2218 and A 2142 (Giovannini \& Feretti 2000), and RXC J1314.4-2515 (Venturi et al. 2007), which do not belong to the two classes above.

\section{Observed scaling relations}

There are several observed correlations for GHs that relate thermal and non-thermal properties of the ICM: those between the radio power at $1.4 \mathrm{GHz}, P_{1.4}$, and the $\mathrm{X}$-ray luminosity, $L_{\mathrm{X}}$, temperature, $T$, and cluster mass, $M$ (e.g., Liang et al. 2000; Feretti 2000; Govoni et al. 2001; Cassano et al. 2006). Additional correlations were also explored between $P_{1.4}$ and the size of GHs, $R_{\mathrm{H}}$, the total cluster mass within $R_{\mathrm{H}}$, and the cluster velocity dispersion (C07). C07 find a trend between $R_{\mathrm{H}}$ and the cluster virial radius, $R_{\mathrm{V}}$, and show that all the other correlations explored so far can be derived by combining the $R_{\mathrm{H}}-R_{\mathrm{V}}$ and $P_{1.4}-R_{\mathrm{H}}$ scalings. This suggests that there are essentially two main scaling relations that carry the leading information on the physics of non-thermal components. The statistical properties of MHs are less explored, indeed few objects are known to possess a $\mathrm{MH}$ and only a trend between $P_{1.4}$ and the maximum power of the cooling flow (estimated as $P_{\mathrm{CF}}=\dot{M} k T / \mu m_{\mathrm{p}}$, Gitti et al. 2004) has been found so far for this class of sources.

In Fig. 1 we show the distribution of $\mathrm{GHs}$ in the $\left(P_{1.4}, L_{\mathrm{X}}\right)^{1}$ plane together with the correlation from Cassano et al. (2006). The MHs are also reported in Fig. 1, where we find that they share the same region of GHs and that a possible $P_{1.4}-L_{\mathrm{X}}$ correlation also exists for MHs and is barely consistent with that of GHs. In Fig. 1 we also report upper limits to the radio power of CCCs without detected MHs taken from the statistical sample of X-ray luminous clusters with deep radio follow up of Venturi et al. (2008). These CCCs without MHs are all relaxed clusters with a central active radio galaxy. Limits were obtained following the approach given in Brunetti et al. (2007, hereafter

\footnotetext{
1 Radio powers that have been measured at different frequencies are converted at $1.4 \mathrm{GHz}$ with a spectral index $\alpha=1.3$, with $S \propto v^{-\alpha}$.
}

Table 1. Sample of galaxy clusters hosting mini radio halos.

\begin{tabular}{lclll}
\hline \hline Name & $z$ & $\begin{array}{l}\log L_{\mathrm{X}} \\
(\mathrm{erg} / \mathrm{s})\end{array}$ & $\begin{array}{l}\log P_{1.4 \mathrm{GHz}} \\
(\mathrm{W} / \mathrm{Hz})\end{array}$ & $\begin{array}{l}\log R_{\mathrm{H}} \\
(\mathrm{kpc})\end{array}$ \\
\hline Perseus (a) & 0.018 & $44.82_{-0.01}^{+0.01}$ & $24.27_{-0.04}^{+0.04}$ & 2.12 \\
A2390 (b) & 0.228 & $45.13_{-0.05}^{+0.05}$ & $24.99_{-0.02}^{+0.02}$ & 2.26 \\
A2626 (c) & 0.060 & $44.03_{-0.05}^{+0.05}$ & $23.36_{-0.10}^{+0.03}$ & 1.85 \\
RX J1347.5-1145 (d) & 0.451 & $45.65_{-0.05}^{+0.05}$ & $25.28_{-0.01}^{+0.01}$ & 2.41 \\
Z7160 (e) & 0.258 & $44.93_{-0.03}^{+0.03}$ & $24.34_{-0.05}^{+0.05}$ & 2.24 \\
RBS 797 (f) & 0.350 & $45.31_{-0.02}^{+0.02}$ & $24.63_{-0.04}^{+0.04}$ & 2.01 \\
\hline
\end{tabular}

References for the radio data in brackets: (a) Sijbring (1993), (b) Bacchi et al. (2003), (c) Gitti et al. (2004), (d) Gitti et al. (2007), (e) Venturi et al. (2008), (f) Gitti et al. (2006), Gitti et al., in prep.

B07) and lie one order of magnitude below the radio power of MHs. Despite the poor statistics, this suggest that MHs may not be common in CCCs.

In Fig. 2 we show the distribution of GHs in the $\left(P_{1.4}, R_{\mathrm{H}}\right)$ plane, where $R_{\mathrm{H}}$ is the radius of the emitting region (as in C07). Following the procedure in $\mathrm{C} 07$, we also derived $R_{\mathrm{H}}$ for MHs from the radio images of these sources. We also find a trend between $P_{1.4}$ and $R_{\mathrm{H}}$ for $\mathrm{MH}$ (Fig. 2) with $P_{1.4}$ rapidly increasing in larger MHs. This trend, however, is not consistent with what is found for GH. The clear separation demonstrates the importance of exploring the distributions of these radio sources in different planes to distinguish between different radio sources and to investigate their physics and origins.

In Figs. 1 and 2 we also report on A 2218, A 2142, and RXC J1314. They are less luminous than GH (Bacchi et al. 2003) and $\mathrm{MH}$ in clusters with similar $L_{\mathrm{X}}$, and are located between $\mathrm{GH}$ and $\mathrm{MH}$ in the $\left(P_{1.4}, R_{\mathrm{H}}\right)$ plane. They are in merging clusters and are smaller (smaller emitting volumes and thus radio powers) than $\mathrm{GH}$, suggesting that they might be $\mathrm{GH}$ at an early evolutionary stage.

\section{Basic interpretation of the observed scalings}

In the case of GHs B07 found that $70 \%$ of clusters in the Venturi et al. (2008) sample are radio quiet, not showing Mpc scale synchrotron radio emission, and that the limits on their radio powers lie one order of magnitude below the correlation followed by clusters with GHs. They discussed that the bi-modality between radio quiet clusters and GH clusters is in line with the re-acceleration scenario, in which turbulence powers up GHs only for a limited period during cluster mergers, and disfavors secondary models that would instead predict GHs that are much more common in galaxy clusters. Despite the poor statistics, MHs in Fig. 1 show a behavior similar to that of GHs in B07. Furthermore, these MHs are characterized by non-relaxed cores, whereas the CCCs without MHs are all relaxed clusters. This might suggest that turbulence connected with merger activity could also play a role in the acceleration of electrons in MHs. More generally, turbulence necessary to trigger MHs could result from the amplification in the cool core of a seed turbulence present in the ICM (Gitti et al. 2002, 2004), could be connected with the gas-sloshing mechanism in CCCs (Mazzotta \& Giacintucci 2007), or could be driven by minor mergers (see also Gitti et al. 2007a) that are common in CCCs with MHs (Sect. 2). Another possibility is that turbulence takes a small fraction of the energy released by the "bubbles" rising from the central AGN, 


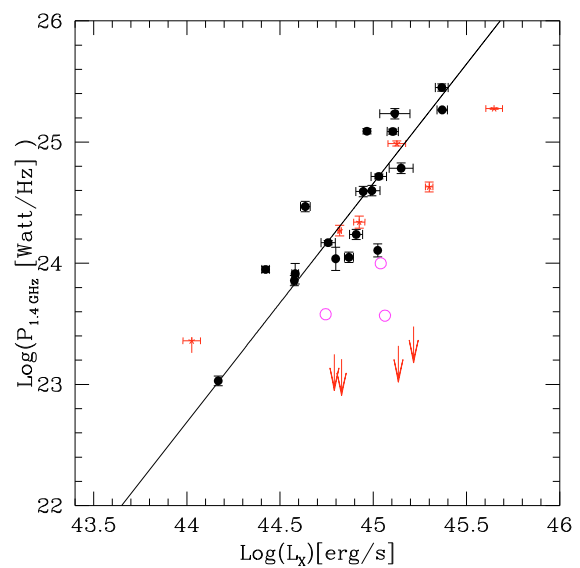

Fig. 1. Radio power at $1.4 \mathrm{GHz}$ versus X-ray luminosity in the [0.1-2.4] keV band of clusters with GHs (black circles), MHs (red asterisks), and small-scale radio emissions (magenta open circles). Arrows are upper limits to the radio power of CCCs without MHs (see text). The black solid line is the best-fit correlation for GHs (from Cassano et al. 2006).

and this can offset the cooling in most clusters (e.g., McNamara $\&$ Nulsen 2007). All $6 \mathrm{MH}$ clusters indeed have an active radio galaxy at their center and 5 have cavities in the X-ray ICM.

A detailed physical modeling of MHs in CCCs is beyond the scope of this letter, but we can derive some basic constraints here on their physical parameters. We find that, although emitting a similar radio power, the radius of MHs is typically a factor $\approx 4$ smaller than that of GHs (see Fig. 2). This implies a synchrotron emissivity for $\mathrm{MHs} \approx 50$ times larger than that of $\mathrm{GHs}^{2}$. Regardless of the origin of the emitting electrons, the ratio between the synchrotron emissivity of MHs $\left(\dot{\varepsilon}_{\mathrm{MH}}\right)$ and of $\mathrm{GH}\left(\dot{\varepsilon}_{\mathrm{GH}}\right)$ can be written as

$\frac{\dot{\varepsilon}_{\mathrm{MH}}}{\dot{\varepsilon}_{\mathrm{GH}}}=\frac{n_{\mathrm{rel}}^{\mathrm{MH}}}{n_{\mathrm{rel}}^{\mathrm{GH}}}\left(\frac{B_{\mathrm{MH}}}{B_{\mathrm{GH}}}\right)^{\alpha+1}$

where $n_{\text {rel }}^{\mathrm{MH}}$ and $B_{\mathrm{MH}}\left(n_{\mathrm{rel}}^{\mathrm{GH}}\right.$ and $\left.B_{\mathrm{GH}}\right)$ are the number density of radio emitting electrons (at the energy needed to emit the observed synchrotron radiation) and the mean value of the magnetic field strength within the $\mathrm{MH}(\mathrm{GH})$, respectively, and $\alpha$ is the radio spectral index of the synchrotron spectrum, which is similar in GHs and MHs ( $\alpha \sim 1.1-1.3$, e.g., Feretti \& Giovannini 2007). The measure of $B$ in the ICM is quite problematic and different methods often give different estimates. Faraday rotation measure studies generally found a few to $10 \mu \mathrm{G}$ in non-CCCs and $\sim 10-30 \mu \mathrm{G}$ in the central region of CCCs (Clarke 2004; Govoni $\&$ Feretti 2004), whereas methods based on inverse Compton emission found from $\sim 0.1$ to $\mu \mathrm{G}$ (Fusco-Femiano et al. 2004; Sanders et al. 2005). There is, however, agreement on the fact that the magnetic field at the center of CCCs is larger than that on the Mpc scale in non-CCCs.

A suitable assumption for the ratio $\left(B_{\mathrm{MH}} / B_{\mathrm{GH}}\right)$ in Eq. (1) (with $B_{\mathrm{MH}} \gg B_{\mathrm{GH}}$ ) allows us to reproduce the observed ratio between the emissivities. However, the difference in terms of $B$ cannot be the only cause of the large synchrotron emissivity in MHs. Indeed a large $B$ in CCCs produces a fast cooling of relativistic electrons due to synchrotron losses (that make $n_{\mathrm{rel}}^{\mathrm{MH}}$ smaller), and this implies the important point that a very efficient mechanism of injection and/or acceleration of relativistic electrons should also be active in MHs.

2 This value should be considered as a lower limit since MHs have also more peaked profiles than GHs.

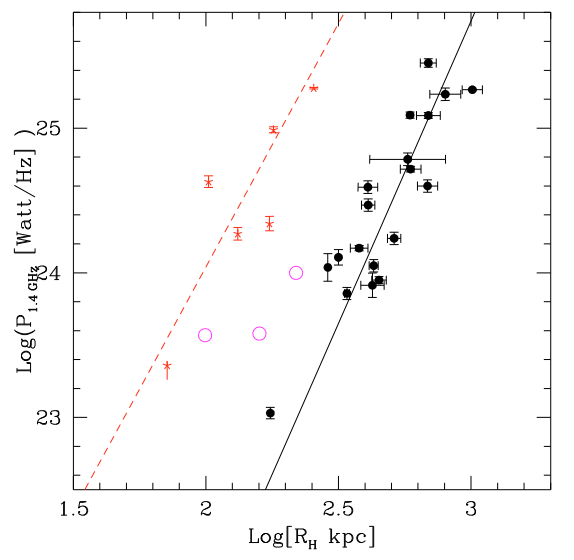

Fig. 2. Radio power at $1.4 \mathrm{GHz}$ versus radio size of GHs (black circles) and MHs (red asterisks), and small-scale radio emissions (magenta open circles). The black solid line and the red dashed line are the best-fit correlations for GHs $\left(P_{1.4} \propto R_{\mathrm{H}}^{4.18}\right.$, from Cassano et al. 2007) and for MHs $\left(P_{1.4} \propto R_{\mathrm{H}}^{3.4}\right)$, respectively.

To quantify this point in a relevant case, we assume that electrons are re-accelerated sporadically by turbulence injected in the emitting region by some process. Electrons are accelerated up to the energy where acceleration is balanced by losses, $m_{\mathrm{e}} c^{2} \gamma_{\mathrm{b}} \propto \chi / \beta$, where $\chi$ is the acceleration efficiency and $\beta=$ $\left(B_{\mathrm{cmb}}^{2}+B^{2}\right)$ accounts for the synchrotron and inverse Compton losses ${ }^{3}$, and a corresponding break forms in the emitted synchrotron spectrum at $v_{\mathrm{b}} \propto B \gamma_{\mathrm{b}}^{2}$. Magnetosonic waves are proposed as possible sources of particle acceleration in the ICM (Cassano \& Brunetti 2005; Brunetti \& Lazarian 2007); and in this case, following C07, the synchrotron emissivity (if the damping of turbulence is dominated by thermal electrons, for $\left.\epsilon_{\text {rel }} / \epsilon_{\text {th }} \ll 1\right)$ is

$\dot{\varepsilon}_{\text {syn }} \propto \dot{\varepsilon}_{\mathrm{t}}\left(\epsilon_{\mathrm{rel}} / \epsilon_{\mathrm{th}}\right) B^{2} T^{1 / 2} \beta^{-1}$

where $\dot{\varepsilon}_{\mathrm{t}}$ is the turbulence injection rate, $\epsilon_{\mathrm{rel}} / \epsilon_{\mathrm{th}}$ the ratio between the energy density of relativistic and thermal particles, and $T$ the cluster temperature. We consider the case in which $\mathrm{GH}$ and $\mathrm{MH}$ have $v_{\mathrm{b}}^{\mathrm{MH}}=f v_{\mathrm{b}}^{\mathrm{GH}}$, and $f=1$ would imply that $\mathrm{GH}$ and $\mathrm{MH}$ have similar spectral index, in line with present observations. From Eqs. (35) and (36) in Cassano \& Brunetti (2005) (in the case $\left.\epsilon_{\mathrm{rel}} / \epsilon_{\mathrm{th}} \ll 1\right) \dot{\varepsilon}_{\mathrm{t}} \propto \beta n_{\mathrm{th}}\left(T v_{\mathrm{b}} / B\right)^{1 / 2}\left(n_{\mathrm{th}}\right.$ is the thermal gas density) and assuming $v_{\mathrm{b}}^{\mathrm{MH}}=f v_{\mathrm{b}}^{\mathrm{GH}}$, one can derive the ratio between the turbulence injection rate in the two populations of sources. A comparison between the energy injected in the form of magnetosonic waves (in unit of the thermal energy, $\epsilon_{\mathrm{t}} / \epsilon_{\mathrm{th}}$ ) in $\mathrm{GH}$ and $\mathrm{MH}$ (assuming $\epsilon_{\mathrm{t}}=\dot{\varepsilon}_{\mathrm{t}} \Delta \tau \approx \dot{\varepsilon}_{\mathrm{t}} R_{\mathrm{H}} / c_{\mathrm{s}}$, with $\Delta \tau$ and $c_{\mathrm{s}}$ being the injection time scale and the sound speed, respectively) is reported in Fig. 3. This figure shows that the energetic request in the case of $\mathrm{MH}$ is comparable to that of $\mathrm{GH}$ provided that the magnetic field in CCCs is not significantly larger than $\approx 10 \mu \mathrm{G}$; in this case, $\epsilon_{\mathrm{t}} / \epsilon_{\mathrm{th}} \sim 0.1-0.3$ is typically required to have $\nu_{\mathrm{b}} \approx$ $1 \mathrm{GHz}$ (Brunetti \& Lazarian 2007).

By combining the expression for $\dot{\varepsilon}_{\mathrm{t}}^{\mathrm{MH}} / \dot{\varepsilon}_{\mathrm{t}}^{\mathrm{GH}}$ with Eq. (2) one finds the ratio between the synchrotron emissivities in $\mathrm{GH}$ and $\mathrm{MH}$ :

$\frac{\dot{\varepsilon}_{\mathrm{syn}}^{\mathrm{MH}}}{\dot{\varepsilon}_{\mathrm{syn}}^{\mathrm{GH}}}=f^{1 / 2}\left(\frac{B_{\mathrm{MH}}}{B_{\mathrm{GH}}}\right)^{3 / 2} \times \frac{\epsilon_{\mathrm{rel}}^{\mathrm{MH}}}{\epsilon_{\mathrm{rel}}^{\mathrm{GH}}}$.

${ }^{3} B_{\mathrm{cmb}} \approx 3.2(1+z)^{2} \mu \mathrm{G}$ is the equivalent magnetic field strength of the cosmic microwave background (CMB) radiation. 


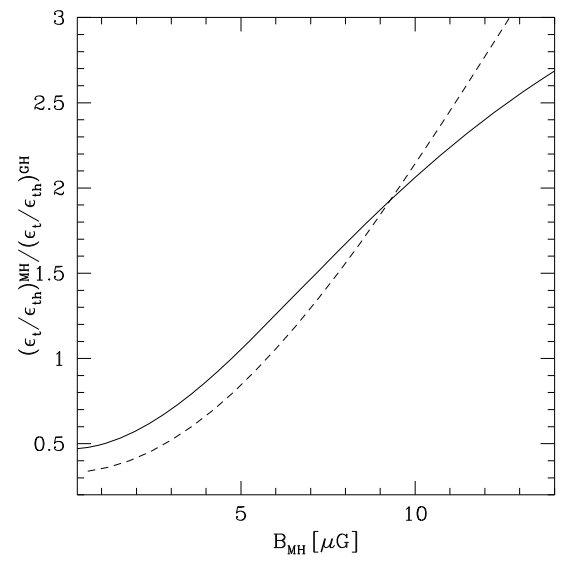

Fig. 3. Ratio between turbulence energy densities of MHs and GHs normalized to the thermal ones as a function of $B_{\mathrm{MH}}$. The calculations are reported for $f \approx 1, z=0.1, T_{\mathrm{GH}} / T_{\mathrm{MH}} \approx 3$ and in the case of $B_{\mathrm{MH}} \approx 3 B_{\mathrm{GH}}$ (solid line) and in the case $B_{\mathrm{MH}} \approx 6 B_{\mathrm{GH}}$ (dashed line).

This implies that to understand the large differences in terms of emissivity $\left(\dot{\varepsilon}_{\text {syn }}^{\mathrm{MH}}>50 \dot{\varepsilon}_{\text {syn }}^{\mathrm{GH}}\right)$ observed between $\mathrm{MH}$ and $\mathrm{GH}$ an extra amount of relativistic (fossil) electrons that take part in the acceleration process in CCCs is necessary $\left(\epsilon_{\mathrm{rel}}^{\mathrm{MH}} / \epsilon_{\mathrm{rel}}^{\mathrm{GH}} \approx 4-10\right.$ assuming $B_{\mathrm{MH}} / B_{\mathrm{GH}} \approx 3-6$ and $f \approx 1$ ). This extra amount of relativistic electrons may be provided naturally by the central radio galaxy or by the p-p collisions (CR protons and thermal protons) in the dense CCCs that may inject efficiently secondary electrons, to be re-accelerated during the activity of $\mathrm{MH}$.

\section{Conclusions}

In this letter we have compared the observed properties of mini radio halos (MHs) and giant radio halos (GHs) in clusters of galaxies. GHs are the most prominent evidence of non-thermal components in the ICM and several correlations between thermal and non-thermal properties have been explored for these sources, including those relating $P_{1.4}$ to $L_{\mathrm{X}}$ and to $R_{\mathrm{H}}$ (e.g., Cassano et al. 2006, 2007; Brunetti et al. 2007). On the other hand, an extensive investigation of the statistical properties of MHs is presently not possible since only a few clusters host wellstudied MHs.

We collected a sample of $\mathrm{MH}$ and compared their behavior with that of GHs in the $\left(P_{1.4}, L_{\mathrm{X}}\right)$ and $\left(P_{1.4}, R_{\mathrm{H}}\right)$ planes. We find that $P_{1.4}-L_{\mathrm{X}}$ and $P_{1.4}-R_{\mathrm{H}}$ trends may also exists for MHs. While in the $\left(P_{1.4}, L_{\mathrm{X}}\right)$ plane $\mathrm{MHs}$ and $\mathrm{GHs}$ share the same region, in the $\left(P_{1.4}, R_{\mathrm{H}}\right)$ plane $\mathrm{MHs}$ do not follow the same correlation of $\mathrm{GHs}$ at smaller radii, but are clearly separated. We find that the typical synchrotron emissivity of MHs is at least 50 times larger than that of GHs. This implies a very efficient mechanism at the origin of the emitting electrons in MHs. For completeness we also consider the few cases of smaller scale emission in nonCC (and without central radio galaxy) merging clusters. These sources are morphologically intermediate between $\mathrm{GH}$ and $\mathrm{MH}$ and may be $\mathrm{GH}$ at some early evolutionary stage.

The distribution in the $\left(P_{1.4}, L_{\mathrm{X}}\right)$ plane of a small sample of CCCs with available radio observations suggests that MHs are not ubiquitous in CCCs, with upper limits for CCCs without diffuse radio emission well below the radio power of MHs in clusters with similar $L_{\mathrm{X}}$. Those CCCs without MHs also appear to be more relaxed than that with MHs. All these findings, if confirmed, would point in favor of sporadic turbulent re-acceleration as the origin of the emitting particles. In addition to the possibilities already explored in the literature (Gitti et al. 2002; Mazzotta \& Giacintucci 2007), minor mergers (see also Gitti et al. 2007a) and/or the central AGN outbursts may contribute to the injection of turbulence in the ICM of CCCs. By adopting this scenario, under the assumption that magnetosonic waves drive the particle acceleration process, we find that the larger synchrotron emissivity of MHs can be explained by assuming that the energy density of the relativistic particles that interact with turbulence is about one order of magnitude higher than in GHs, and that this does not necessarily imply a larger amount of turbulence in MHs. The extra amount of relativistic particles in these sources may be provided by the central cluster galaxy or by secondary electrons injected in the dense cool core region.

Acknowledgements. We thank G. Setti, S. Giacintucci, T. Venturi, and the anonymous referee for useful comments. This work is partially supported by grants PRIN-INAF2005, and ASI-INAF I/088/06/0.

\section{References}

Allen, S. W., Ettori, S., \& Fabian, A. C. 2001, MNRAS, 324, 877 Allen, S. W., Schmidt, R. W., \& Fabian, A. C. 2002, MNRAS, 335, 256 Bacchi, M., Feretti, L., Giovannini, G., \& Govoni, F. 2003, A\&A, 400, 465 Blasi, P., \& Colafrancesco, S. 1999, APh, 12, 169

Brunetti, G. 2003, in Matter and Energy in Clusters of Galaxies, ed. S. Bowyer, \& C.-Y. Hwang, ASP Conf. Ser., 301, 349

Brunetti, G., \& Lazarian, A. 2007, MNRAS, 378, 245

Brunetti, G., Setti, G., Feretti, L., \& Giovannini, G. 2001, MNRAS, 320, 365

Brunetti, G., Venturi, T., Dallacasa, D., et al. 2007, ApJ, 670, L5

Buote, D. A. 2001, ApJ, 553, 15

Cassano, R., \& Brunetti, G. 2005, MNRAS, 357, 1313

Cassano, R., Brunetti, G., \& Setti, G. 2006, MNRAS, 369, 1577

Cassano, R., Brunetti, G., Setti, G., et al. 2007, MNRAS, 378, 1565

Cassano, R., Brunetti, G., Venturi, T., et al. 2008, A\&A, 480, 687

Churazov, E., Forman, W., Jones, C., et al. 2004, MNRAS, 347, 29

Clarke, T. E. 2004, JKAS, 37, 337

Feretti, L. 2000, Invited review at IAU 199, The Universe at Low Radio Frequencies, in Pune, India, 1999

Feretti, L. 2005, in X-Ray and Radio Connections, published electronically by NRAO, ed. L. O. Sjouwerman, \& K. K. Dyer

Feretti, L., \& Giovannini, G. 2007, published in Springer Lecture Notes in Physics, Panchromatic View of Clusters of Galaxies and the Large-Scale Structure, ed. M. Plionis, O. Lopez-Cruz, \& D. Hughes

Ferrari, F., Govoni, F., Schindler, S., et al. 2008, SSRv, 134, 93

Fusco-Femiano, R., Orlandini, M., Brunetti, G., et al. 2004, ApJ, 602, 73

Fujita, Y., Kohri, K., Yamazaki, R., \& Kino, M. 2007, ApJ, 663, L61

Giacintucci, S., Venturi, T., Brunetti, G., et al. 2005, A\&A, 440, 867

Giovannini, G., Tordi, M., \& Feretti, L. 1999, NewA, 4, 141

Gitti, M., \& Schindler, S. 2004, A\&A, 427, L9

Gitti, M., Brunetti, G., \& Setti, G. 2002, A\&A, 386, 456

Gitti, M., Brunetti, G., Feretti, L., \& Setti, G. 2004, A\&A, 417, 1

Gitti, M., Feretti, L., \& Schindler, S. 2006, A\&A, 448, 853

Gitti, M., Ferrari, C., Domainko, W., et al. 2007a, A\&A, 470, L25

Gitti, M., Piffaretti, R., \& Schindler, S. 2007b, A\&A, 472, 383

Govoni, F., \& Feretti, L. 2004, Int. J. Mod. Phys. D, 13, 1549

Govoni, F., Feretti, L., Giovannini, G., et al. 2001, A\&A, 376, 803

Kempner, J. C., \& Sarazin, C. L. 2001, ApJ, 548, 639

Liang, H., Hunstead, R. W., Birkinshaw, M., \& Andreani, P. 2000, ApJ, 544, 686 Mazzotta, P., \& Giacintucci, S. 2008, ApJ, 675, L9

McNamara, B. R., \& Nulsen, P. E. J. 2007, ARA\&A, 45, 117

Peterson, J., \& Fabian, A. C. 2006, Phys. Rep., 427, 1

Petrosian, V. 2001, ApJ, 557, 560

Pfrommer, C., \& Enßlin, T. A. 2004, A\&A, 413, 17

Sanders, J. S., Fabian, A. C., \& Dunn, R. J. H. 2005, MNRAS, 360, 133

Schuecker, P., Böhringer, H., Reiprich, T. H., \& Feretti, L. 2001, A\&A, 378, 408

Sijbring, D. 1993, A radio Continuum and HI Line Study of the Perseus Cluster, Ph.D. Thesis, Groningen

Venturi, T., Bardelli, S., Dallacasa, D., et al. 2003, A\&A, 402, 913

Venturi, T., Giacintucci, S., Brunetti, G., et al. 2007, A\&A, 463, 937

Venturi, T., Giacintucci, S., Dallacasa, D., et al. 2008, A\&A, 484, 327

Wong, K.-W., Sarazin, C. L., Blanton, E. L., \& Reiprich, T. H. 2007, AJ, in press [arXiv: 0803.1680] 